\title{
Crossroads of seeing: about layers in painting and superimposition in Augmented Reality
}

\author{
Manuel van der Veen ${ }^{1}$
}

Received: 31 July 2019 / Accepted: 18 August 2020 / Published online: 26 September 2020

(c) The Author(s) 2020

\begin{abstract}
Augmented Reality (AR) is itself a technology in which two ways of seeing are crossed. Our field of vision is thereby superimposed with digital information and images. But before this, the real environment is already perceived by machine seeing, it is redoubled by a 3D-model, scanned, located and linked. In this brief investigation, I will face the way of seeing in AR with traditional procedures, like 'trompe-l'œil' and the so-called 'velo', to distinguish between what remains classic and what has changed. It is important to examine this as layering, because it is a very thin stack of techniques, technology, materials and media, we seek to watch through. Subsequently, I shall analyze a painting of the contemporary artist Laura Owens in which both ways are crossed, the traditional one and the one concerning AR.
\end{abstract}

Keywords Augmented Reality $\cdot($ Digital) layering $\cdot$ Stack $\cdot$ Superimposition $\cdot$ Transparency $\cdot$ Trompe-l'œil $\cdot$ Velo (veil)

\section{Introduction}

The field of vision in Augmented Reality (AR) challenges our way of seeing by registering digital images and objects onto the real environment. Sometimes these images and objects emerge as registered and sometimes, they blur the boundaries between the digital and the real area. It is my approach to face AR with its cognates in art history to sort out the specific strategies and procedures of layering. It is not the goal to prove some continuous development from ancient illusion techniques to newer technologies. Rather, the new should be divorced from the already known to examine our current way of seeing in AR more profoundly. The view through the glasses of AR enables a new perspective onto the tradition. The technology merges various techniques and procedures; in particular, it crosses two ways of seeing: our view through the eyes and that of machine seeing. The latter processes the data, received by the sensors and cameras, within our field of vision to calculate a hybrid view. However, this leads into a double blindness, as each participant is blind to the other for a certain extent of the way.

Manuel van der Veen

M.vanderVeen@me.com

1 State Academy of Fine Arts Karlsruhe, Karlsruhe, Germany
In general, AR may be defined as an operation of superimposition. It overlays the real environment and one has to perceive them together. For this, there are various scenarios in earlier procedures. For example, Filippo Brunelleschi's two experiments in front of the Baptistery San Giovanni and at the Piazza della Signoria, placing cut-out paintings surrounded by the movement of clouds or the living city. We must also think of the phantasmagoria, within which ghosts are projected into the real space and in real time (Elcott 2016). Furthermore, the schüfftan-process, perhaps less common, also establishes an interplay of real fragments with illusionistic complements. Finally, there are analogue panoramic boards that provide information about the location, at the location. All of these are relatives which are not the same, but are particularly suited to highlight differences.

In this text, I would like to focus particularly on the layer of superimposition itself. It is placed between an imaginary and a real interface. To look at this at least semitransparent layer I suggest to investigate Leon Battista Alberti's "howto-do-it apparatus", the so-called 'velo' (veil). 
Fig. 1 Device for perspectival drawing of a landscape. Anonym, 1710. PD-Art/PDold-100=/1810/?; PD-US

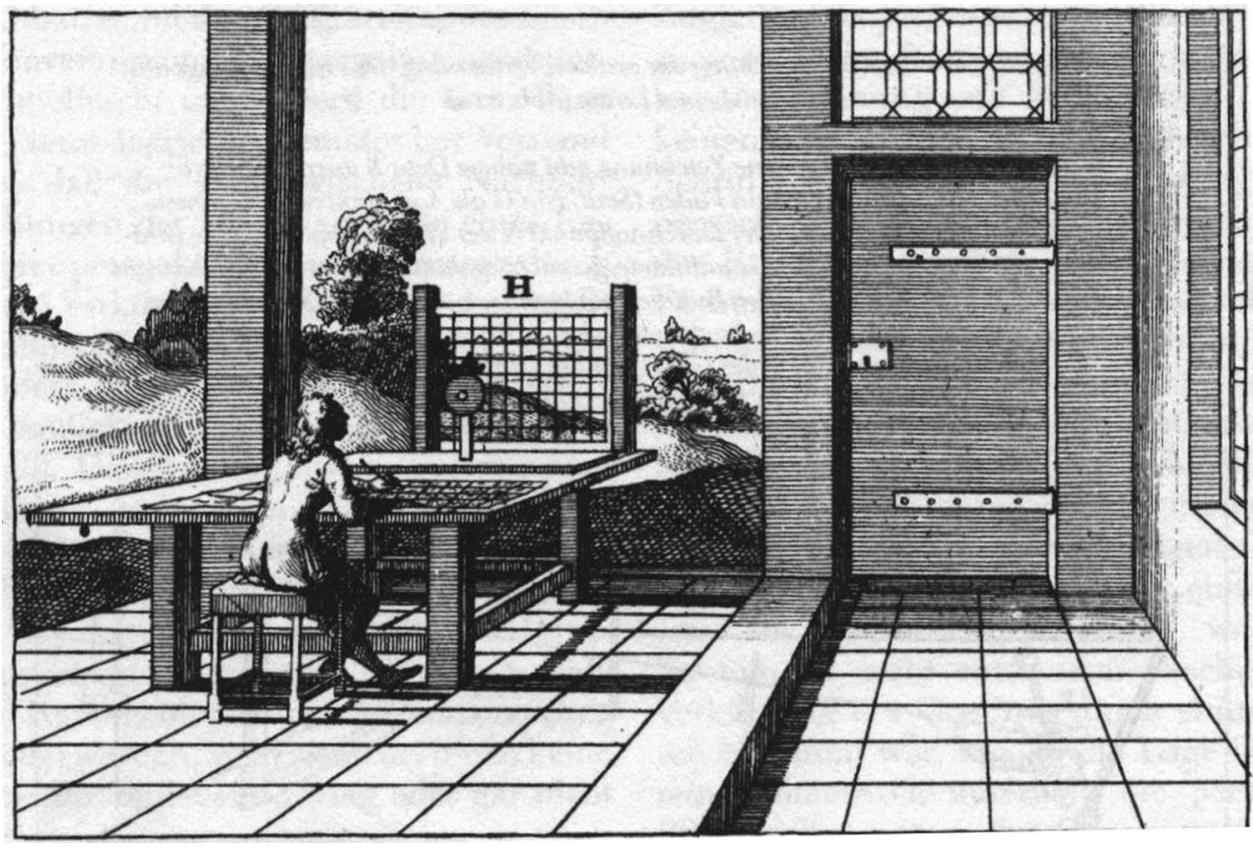

\section{Metaphor and apparatus: about the 'velo' as a layering procedure}

To analyze the 'velo' as an apparatus depends on the observation that the device itself establishes a way of seeing. As noted above, AR tends to blur the boundaries between digital images and the real surroundings. In contrast to the career of immersion into the image, which is generally associated with virtual reality, in AR immersive images are placed. Their status as images is covered to appear as a part of reality, which connects the current view to the traditional procedure of 'trompe-l'œil'. However, it has not yet been decided whether AR will pursue an all over camouflage. My research project 'Augmented Reality. Trompe l'oeil and Relief as Technique and Theory', of which the considerations here form an excerpt, suggests to describe 'trompe-l'œil' and sculptural relief as immersive and emersive ${ }^{1}$ images. Since the 'velo' provides a layer to think the technological implications of AR and at the same time plays an important part in the interpretation of 'trompe-l'œil', it is particularly suitable for the following investigation.

In 1435 Alberti wrote in his treatise on painting and perspective 'De pictura' that the rectangular frame of paintings should be seen as an open window ('aperta finestra'). This very well-known section from 'De pictura' also carries well-known difficulties. First, the solid and opaque surface of the canvas is denied. And second, what is seen through a window is the here and now; a painting of the fifteenth and

\footnotetext{
1 Thanks to a productive conversation with Matthias Bruhn, about emersion and emersiv images, the parallel to the procedure of sculptural relief could be drawn.
}

sixteenth centuries instead usually shows a somewhere and a sometime else of an 'istoria'. The extensive discussion of the window metaphor cannot be pursued further here. For now, it seems more interesting to look up another passage from Alberti's treatise in book two. Here, he does not only describe a metaphor, but also an actual apparatus, a device. This apparatus stretches out a semi-transparent cloth with a grid of threads as a layer between the artist and the motif (Fig. 1). It is precisely this cloth, which I proposed as a relative of the information and object layer, that we are dealing with in AR.

The technology circulates as a procedure, which places figures in the environment, as in the popular application 'Pokémon Go' (Niantic). But even before a figure, an image or an object occupies our field of vision, the technology perceives the environment, doubles or multiplies the pair of eyes through cameras and sensors to set up a (almost invisible) layer through which we perceive the environment. It might be helpful to quote Edmund Husserls ternary image theory, which consists of three layers of seeing: physical image_image object-image subject (Husserl 2005: 20). The physical image is for example the carrier, the physical support. The images of AR and of 'trompe-l'œil' are problematic as such because they seem to have no carrier. ${ }^{2}$

Due to that lost carrier, the layer of projection and the performance of the machine are suppressed. And with them, the place is blurred where the two ways of seeing, of

\footnotetext{
2 The missed physical image in Augmented Reality in relation to Husserl's image theory was mentioned during a presentation by Stephan Günzel in Weimar, Germany (Das Diorama: Durch...Denken) called 'Augmented Reality: Zur (In)Transparenz des Bildes'.
} 
machine and body, are crossed. Everything that becomes visible through the glasses of AR is already processed. Husserl's image theory is based on the difference between image and environment, the difference that is at stake in AR and 'trompe-l'œil'. Thus, for Husserl, the image manifests itself in a conflict and this conflict is not caused by the realistic depiction (Husserl 2005: 51): "The appearance belonging to the image object is distinguished in one point from the normal perceptual appearance. This is an essential point that makes it impossible for us to view the appearance belonging to the image object as a normal perception: it bears within itself the characteristic of unreality, of conflict with the actual present. The perception of the surroundings, the perception in which the actual present becomes constituted for us, continues on through the frame and then signifies 'printed paper' or 'painted canvas.." If the carrier is lost, the conflict with the actual present disappears, which in turn leads to an uncertain image perception in AR.

The motivation to look out for the carrier is not based on its status of being lost or hidden, which would end up in chasing a deception. Rather, it is due to the observation that the carrier is shifted instead. A 'trompe-l'œil' occupies a classical support, but only to be its alienation, because the procedure stacks other depicted supports onto the image support (e.g. planks of wood, papers, canvases, etc.). With each depiction, the physical support is pushed forward piece by piece (Fig. 2). Therefore, we could record that the carrier is suppressed by both, its multiplication and by its disappearance.

In AR a carrier also exists as a (semi-transparent) display in the optical-see-through technique, which directs the image to the eye and as a touchable screen in the video-see-through technique of handheld devices. To call it see-through thus ties the technique directly with that of perspective as a seeing through. But the carrier is already slipped. On one hand, the video-see-through technique shows both, the actual surroundings behind the screen and the superimposition on the screen. In the optical-see-through, on the other hand, the carrier is placed directly in front of the eyes to appear imaginarily over there, in the middle of the surroundings. The carrier is, therefore, no longer a background, but rather shifted forward. In 'trompe-l'œil' and AR, the three levels of image perception collapse: the environment seems to become the carrier and finally, determines image object and image subject.

In summary, the physicality of the carrier, as well as the status as an image, become instable. However, the fact that the carrier is shifted, multiplied or transparent changes the way of seeing beyond a mere deception. In the following, I would like to introduce the 'velo' as a layer to think the lost carrier as a literal interface. Not as a background-but as a layer between the observer and the surroundings, which also requires a shift in perception. In AR we do not just look at

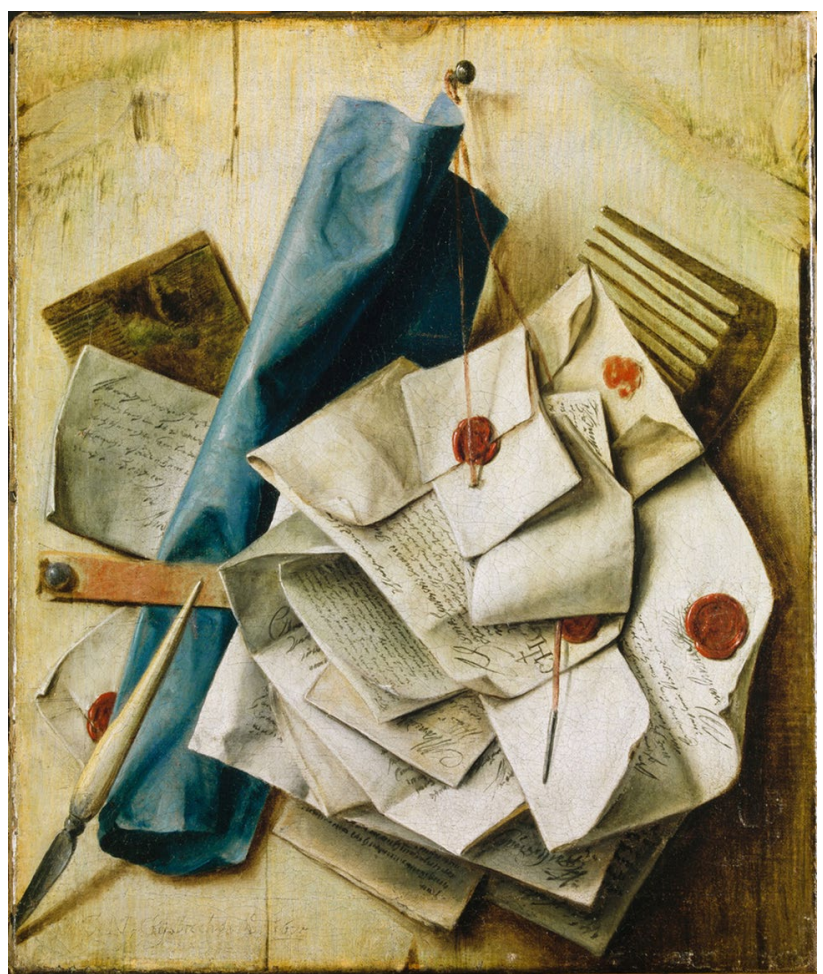

Fig. 2 Cornelis Norbertus Gysbrechts, Quodlibet or "Vanitas-Stillleben”, 1675. oil on canvas. $41 \times 34,5 \mathrm{~cm}$. Wallraf-Richartz-Museum \& Fondation Corboud, Köln, Inv.-Nr. WRM 2828. Copyright: Rheinisches Bildarchiv Köln, Rolf Zimmermann, rba_c011283. https://www. kulturelles-erbe-koeln.de/documents/obj/05011135

an image, we also look at the world through a layer. A layer which organizes the complex of world and image and thus our way of seeing. With the 'velo', different ways of this organization are to be worked out. The layer of the 'velo' is first constitutive as a translation function, then it is perceived with its own materiality, only to finally give up its materiality again and become an operation of structuring.

\subsection{The 'velo': a layer for translation}

The 'velo' is a semi-transparent cloth with a gridded surface through which one can see into depths. Decisive for the change in mind is the transition from a metaphor of an open window to a real studio tool. The framework continues inside the 'velo' and leads to the crucial difference to the metaphor, which is highlighted by Anne Friedberg: "but while Alberti suggested the rectangular frame and planar surface of a metaphoric 'window'; as a device for geometric calculation, his 'velo' did not require the calculation of orthogonals and vanishing points. It was, instead, dependent solely on its frame and its inset quadrants as a device to 'map'; the three-dimensional world onto a two-dimensional plane." (Friedberg 2006: 38). 
This mapping procedure is a translation and not a construction. If one looks through the 'velo', the image is already in the frame or better, within the many small frames - it just needs to be transferred onto the paper. Round bodies and their relief are already present on the surface, likewise to a projection. While turning the gaze back and forth, the artist translates what he sees, frame by frame onto the similar grid on the sheet of paper, watching the outsidenot to orientate in the landscape but rather on the drawing. Emmanuel Alloa describes this translation, in reference to the 'velo', as a taming of the mobile. Contrary to this, he emphasizes the greater mobility of the individual elements in the grid, which causes the subdivision of the objects (Alloa 2011: 156). This description includes an important difference to AR, which does not translate an image onto a sheet of paper. It translates the actual view in real time, by superimposing data, also in real time-with that, it is rather an unleashing of the mobile. A popular effect of AR is to translate fixed images into moving ones. In doing so, these images are superimposed by themselves, but in motion.

\subsection{The 'velo': a layer with its own materiality}

Regarding the translation function, it must be concluded that through the 'velo' only what is in situ can be perceived, what actually is placed behind the frame. Hence, behind that surface a fiction is impossible-but on the surface it can be reintroduced. 'Trompe-l'œil' as a procedure is related to the 'velo' in making its materiality visible and with this, a further function can be assigned to the layer. A 'trompel'œil' usually starts with a redoubling of the image carrier. Thus, it frees itself from the Albertian window and begins to approach the object, to the opacity of the canvas itself. Sybille Ebert-Schifferer shows this approach in her text 'Der Durchblick und sein Gegenteil'. For her, the 'velo' is a projection surface and this surface becomes a membrane which, although transparent, is a material separation between the space of the viewer and that of the picture (Ebert-Schifferer 2016: 16). The 'velo' refers to both the classical representation and to the object status of the painting, thus it creates a hybrid view. For 'trompe-l'œil', it is important to appear as an object at first, not as a picture. Therefore, it uses different techniques to make its own materiality credible. Hence, the space of 'trompe-l'œil' is extremely flat. According to Ebert-Schifferer, in 'trompe-1'œil', the membrane of the 'velo' stretches slightly forwards and backwards. She imagines how artists have attached notes onto the 'velo' or that a fly came to rest on it. If the 'velo' is understood as a material layer, then new possibilities show up: first, one may use the space in front of the 'velo' and second, the motif behind it can be superimposed. It is precisely this space in front of the layer, that makes 'trompe-l'œils' as objects so believable and which connects the traditional procedure with AR. As a material layer, it becomes an object of use-to pin something into it or to write on it.

\subsection{The 'velo': a layer as a way of seeing}

Above, we have noted that the grid of the 'velo' causes the subdivision of the objects. Reading between the lines, one could say the grid provides an organization. The individual quadrants are elements of a relationship — a relationship that can be changed. From a translation function to the visualization and use of its own materiality, the path of the 'velo' branches out even further. It slowly leaves the place of the studio to expand into the everyday perception. To follow this path, it is illuminating to look at a few didactic illustrations, which are designed close to the actual field of vision. A trace for this transfer is found in the books mentioned by Samuel Y. Edgerton. He examines technical and scientific treatises, which have been printed since 1520 with numerous illustrations. In these books, word and image build a unity as never seen before, which Edgerton attributes to the imaginary grid celebrating its career at that time in cartography. He emphasizes that it was the 'velo' that educated artists to see the underlying geometry in nature. This didactics spread, therefore, all educated people were able to think this invisible, but indispensable grid that underlies every picture (Edgerton 2004: 181). Due to the technical, didactic and practical advantages, a unique image form developed, which Edgerton calls an 'incongruent sign convention'. This is described as a superimposition of otherwise illusionistic scenes by flat, abstract geometric diagrams placed directly above them to explain the underlying mathematical principle. As shown in Fig. 3, the superimposition marks a perfect workflow. And if we look closely, it is recognizable that there is more than one layer. The second angle is place between the two figures, the layer is multiplicated and shifted into space. The practical component of the grid was discovered from the autodidactic craftsmen-technicians. A hybrid image was the result of that incongruent sign convention-as if one depicts something in depth through the grid, to draw afterwards on the 'velo' itself, which means preserving the 'velo' and registering it into the image. The gridded layer as interface was used to add constructively specific information. These technique books invented increasingly incongruent drawing conventions that move further and further away from the mere illusionistic representation of perspective: they duplicate objects several times in one picture, mix perspectives and explode assembly drawings. To see the underlying geometry of nature meant, being able to depict an object unnaturally from different perspectives within one image and without any logical separations. The objects are dismantled, labeled and didactically prepared, but placed in a natural landscape. To illustrate this, Figs. 3 and 4 show how the superimposition in AR is live instead, but the similarity 
Fig. 3 Woodcut from Cesare Cesarianos edition of Vitruvius, 1521. Collection Metropolitan Museum of Art. Creative Commons CC0 1.0 Universal Public Domain Dedication
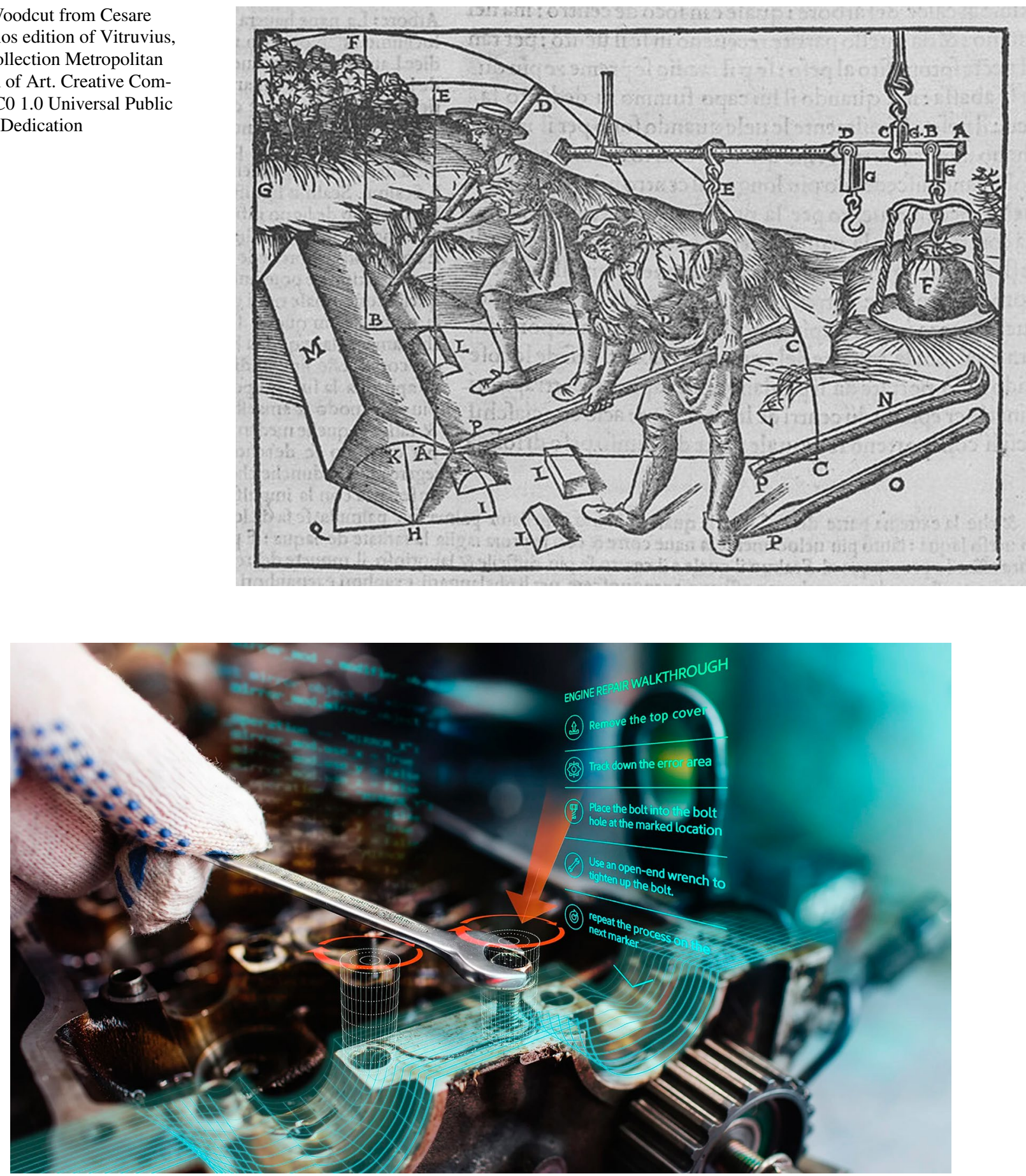

Fig. 4 Application based animation by RE'FLECT for engineering, repair concept https://www.re-flekt.com/hubfs/REFLEKTONE_RepairConc ept_1920x1080.jpg?hsLang=de

is still quite recognizable. An engineer's field of vision (and with this live guidance, certain competencies become more irrelevant) is overlaid with a workflow that both presents an order and anticipates an action. What we see is directly translated into an understandable view, a program to follow, for more efficiency movements and for learning by doing.
The surroundings are visually redoubled by an animation and this animation can be cut up and rearranged. Furthermore, the superimposition by textual marking itself already generates a structure for the perceived.

In short, the 'velo' changed a way of seeing. Its material semitransparency is expressed in the theoretical and 
practical ambivalence of the apparatus. The ambivalence of perspective painting (to have a flat surface, but depict depth) - is not concealed by the 'velo', but rather exposed. Represent depth and draw on the surface. Ultimately, it is not about switching between the two views: both are to be kept in the same field of vision. Starting as a translation function in the studio, its own material structure becomes more and more visible, as if the artists could not ignore the field of vision and the materiality of this tool in the working space. In the end, the interface of the 'velo' is transferred onto thinking. One begins to take the invisible layer as a structuring operation. What is seen through the layer is made more understandable on the layer.

For AR, the translation function, the materiality of the layer, as well as the operation of structuring are decisive. In the first place, there is always a translation function. For the current AR applications on handheld devices, the environment is translated into a $2 \mathrm{D}$ video image which is directly superimposed. 'Head-Mounted Displays' (HMDs), like the 'HoloLens 2', recognize the real environment by spatial mapping and translate it to a live 3D model. What also happens in the optical-see-through technique occurs explicitly in the handheld device. The translation function, the reduction to a flat plane, is augmented to an operation of structuring. By superimposing, the objects and the data are compressed into a flat unit of meaning. Before we look at different opportunities how this unit of meaning could be organized, a further difference should be marked.

In AR the translation of the space is not done by the artist, but by machine vision. Viewers are blind to this process. For example, the world is perceived by the cameras and sensors via spatial mapping, so that objects can be placed credibly in the surroundings. In some applications a grid is animated that spreads over the environment, following its ups and downs, which in turn is only a representation of machine vision, since there is no direct communication between the code and the perception. Within the machine vision works a program, which stipulates our point of view, what is the meaning of program (in Greek: 'pro-graphein'). Katja Glaser and Jens Schröter point out that augmentation describes a program of efficiency, functionalization and optimization. And with AR, this program also inscribes itself into its practices and its field of vision (Glaser and Schröter 2013: 44). Without a carrier the images do not appear as programmed, what one sees is just the representation of the computed surroundings.

Ultimately, both ways of seeing are blind to each other for a certain extent of the way-our field of vision is programmed, but our perception also includes aspects that are beyond the reach of the sensors. The glasses of AR can also provide what is seen and with that the viewers are able to inscribe themselves into the world to program it.

\section{Bundle, loose stack, and heap as models of layering}

In $\mathrm{AR}$, the material carrier is a semi-transparent surface which is slightly darkened. Images projected onto it appear as if they were on site by adjusting their size to the depth of the space. It is as if the real surface of projection itself is projected and extended into the room, exactly this layer itself is sometimes depicted in the field of vision —at least as a pinboard or interface like those in the application 'Spatial' by 'Hololens $2{ }^{\prime} .{ }^{3}$ This makes it possible to place something in front and behind this layer. The imaginary surface can also be multiplied, thereby the individual layers overlap each other and suggest space. Ultimately, this layer is superimposed with information, pictures and objects relating to what can be seen through the semi-transparent surface.

Translation, materiality and structuring operations are inscribed as meta-levels into the grid of the 'velo'. Therefore, I would like to describe the layering process as a stack of these different functions and operations. A stack is characterized by the fact that different levels can be gathered in one place as well as it shares the hybrid status between theoretical and practical characteristics. For this, a stack works also transformatively - it gathers individual, mostly flat elements (for example sheets of paper as in 'trompe-l'œil'), brings them into a common relationship and generates space. In theory, a stack assembles different levels of autonomous functions, but in superpositions, it is a passageway through all of these functions. The view through the glasses of AR is a view through a stack of layers, both literally and metaphorically. A thin stack of different technologies, techniques, media, materials, functions and operations. With the 'velo', the layers of these stack could be bundled between the operation of translation and the function of structuring.

Additionally, a stack contains an intensive aesthetic potential, which presents a unique way of seeing. A stack oscillates between horizontal and vertical. It determines the space of 'trompe-l'œil', the computer desktop and AR. The use of a stack shifts from the desk to the desktop, as Friedberg notes: "The user would manipulate from a position as if in front and also above [...] 'desktops' that defy gravity and transform the horizontal desk into a vertical surface with an array of possible documents and applications: 'icons' that represent objects or, more exactly, object-oriented tasks." (Friedberg 2006: 226). This is crucial in AR-due to the

\footnotetext{
3 To see the animation adequate, please watch the demonstration video at 9:00 min. https://www.youtube.com/watch?v=uIHPPtPBgH $\mathrm{k}$.
} 
better legibility the text is usually set up parallel to the viewers own field of vision. ${ }^{4}$

In 'trompe-1'œil' papers are stacked to leave the surface minimally behind, thereby different layers are visible at the same time. What we see is a stack of sense-fragments, of text-quotations and picture examples which come out towards the viewer (Fig. 2). With that, a stack piles up flat units into something three-dimensional transforming the work of art into an everyday object at the same time. A transformation that encompasses the core of the 'trompel'œil'. Every etching, drawing or text bundled with a ribbon in 'trompe-l'œil', turns into something to use instead of something to look at. Wade Guyton organized an exhibition at the Aspen Art Museum in 2017. He stacked his paintings on the wall, which can be seen as a typical studio situation. Isabelle Graw mentioned they would become a sculpture. The consequence- they cannot longer be experienced aesthetically, instead they have been transformed into a product that can be packaged, exchanged, and traded (Graw 2017: 238). Usually, a stack is bound to gravity, but on screen and in AR the individual layers can be vertically aligned and may appear semi-transparent. In this way, they create a linked image together with the background. In AR, these layers additionally refer to what is visible in the surroundings. Above, I drew a few parallels between the 'velo', 'trompel'œil' and AR. Within the following, I present two different ways in which the stack of layers transform our seeing in the named procedures, beginning with 'trompe-l'œil' to switch to AR. Both procedures model an interplay of different layers, because they are visible simultaneously. Subsequently, I will propose a third possibility, a thought experiment- the concept of the heap, to confront order with chaos.

\subsection{The stack as bundle}

A painterly reflection as well as the current technological one might be examined through the layers extending into depth. The surface of 'trompe-l'œil' can be indicated by cracks and fissures. They refer to an aging process and to a fragility of the specific materials as well as to a deeper level underneath. This allows the viewer to see different layers at the same time. However, the cracks are not placed by chance. In a text about the broken glass in 'trompe-l'œils', Monika Wagner shifts the focus of attention to the materiality, actually depicted through cracks and their structural function as a comment. She also emphasizes the significance of the 'velo' as a medium of flatness. As the 'velo' helps to translate the

\footnotetext{
${ }^{4}$ In his book Cultural Techniques Bernhard Siegert examines the 'trompe-l'œil' as a conflict between two cultural techniques, gazing and reading (Siegert 2015: 164-191). Which also refers to the verticality and the horizontality of a picture.
}

space into the flatness of the picture, the broken and thus visible glass ties the illusory space to the surface (Wagner 2010: 41). What is far apart in reality can thus be connected in the flatness. In other words, a reference is created, because two different things seem to be on the same layer. Using glass, different patterns beside a squaring are possible. This example is about the transparent materiality of glass, the very glass that is the carrier for the projection in AR. ${ }^{5}$ The glass as a carrier of information and as a transparent layer between the observers and the image. The broken glass in the painting of Laurent Dabos about 1808 becomes visible (Fig. 5). It thus provides both, protection (of the underlying layer) and visibility (of the content below). In case of a selfaware positioning of these cracks, it is possible to organize the image through the cracks. Highlighting specific areas on it and making other less clear. Thanks to the transparency of the glass it is possible to superimpose the annotated image with the visual comment, without erasing the image (Wagner 2010: 45). Both share the same field of vision and yet not the same depicted layer. However, it only achieves this through its material-specific properties. The 'trompe-l'œil' of Laurent Dabos shows a stack as well as a broken glass. The origin of the cracks is the text, from which different lines link the individual pictures and figures. The figures' view, highlighted, because it is free of glass, is as sharp as the edges.

The 'trompe-l'œil' thus found an extremely specific layer structure: a layer structure in which the individual layers simultaneously remain in the area of the visible. On the solid, wooden surface are various images and texts arranged, superimposed with a broken glass. These three functionally different layers are bundled into one fixed unit. The unity of that bundle is constituted on one hand by the everyday object of the picture in the frame and on the other hand by the fact that all layers are structurally related to each other. ${ }^{6}$ Finally, the layer of the glass, if it is perceived, decides how we have to read the lower levels.

\subsection{The stack as a loose stack}

The possibilities of AR are based on similar strategies as 'trompe-l'œil', which allow to illuminate and expand each other. The things that in reality are far apart can thus be compressed to the same layer with its superimposition. It is the challenge to bundle the information superimposed

\footnotetext{
$\overline{5}$ Referring to precisely these fractures and cracks, AR often animates a wall breakthrough to allow fictitious elements to break into the real space. This emphasizes the materiality of the wall.

6 Thanks to a discussion with Carolin Meister, the concept of the bundle could be worked out as a fixed stack in which the two outer layers hold an in-between together.
} 


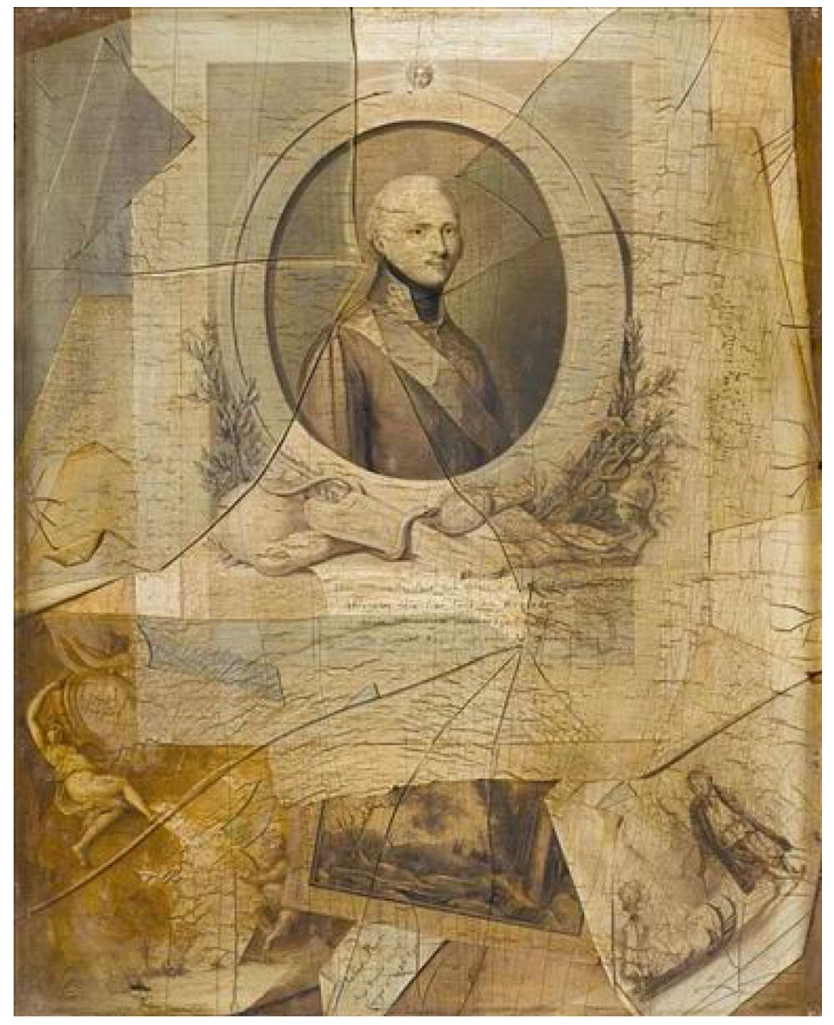

Fig. 5 Laurent Dabos, trompe-l'œil with print of tsar Alexander I of Russia, together with other prints and drawings behind a broken pain of glass, ca. 1808. oil on panel $63,5 \times 50,5 \mathrm{~cm}$. PD-Art/PDold- $100=/ 1835 /$ France; PD-US

on the glass with the related object as tightly as possible. To make this clear, although the arrangement in 'trompel'œil' and in AR seems similar, AR does not present a fixed bundle. Therefore, it could be described as a loose stack in which every layer is able to change its positions immediately, without a reasonable cause. With reference to a museum related device developed by the ArLab Weimar, Oliver Fahle describes the possibilities of the technology and how it changes the concept of the image. Instead of the common term of immersion, usually referring to virtual reality, Fahle explains the technique of AR as participation. It creates a view on another visual layer of the same image (Fahle 2006: 93). He applies this to the specific constructions of Arlab, in which earlier stages of the same painting are projected onto its present layer, thereby the chronological succession is less visible. Decisive for the argumentation is the view onto other layers of the same picture. According to Fahle, the picture is thus augmented by a visual halo, which, however, does occupy the picture itself. The final layer is confronted with earlier stages and information, therefore the pictorial event intervenes in the one unchangeable work and mediates between the one and the many (Fahle 2006: 95). The previously invisible pre-stages now participate with the visible original. The chronological order can be reversed and restacked. The stability of the final work is weakened, without actually being transformed. It shows up as a layer-network that constantly creates new references and thus evokes a shift in mind. The work of art itself is a shift in mind, but the participation of different layers allows to think about layering, temporarily as well as spatially, and to reconsider the final work.

\subsection{The stack as a heap}

I would like to stress a third possibility of a stack to undermine the impression that it is always about a comprehensible system of layering. What I have in mind is a stack fallen down from the desk, which is scattered all over the ground. This kind of layering is a heap, a random arrangement of data and images. The paradox of the heap is that its structure is not recognizable and furthermore, the heap itself cannot be determined. One cannot define how many elements comprise a heap and yet you know that it will remain a heap if you remove some elements. AR is, therefore, also capable of superimposing the field of vision with layers in such a way that it appears to be filled up. The superimposition is that rich in number, that one cannot see what is superimposed: a data heap which collects information from all around to tear the field of vision into pieces.

The possibility of accumulation deprives the stack of its stability in several aspects, firstly, because it introduces disorder and secondly, because it shows that each augmentation includes a reduction - any superimposition, no matter how transparent, carries the possibility of a concealment. With the concept of the heap, the question of the limits of superimposition comes into view. AR aims to filter the diversity of reality for more efficient use by linking specific information to objects. For now, as one opportunity, the infinite diversity of the world and the huge data heap of possible links collide. A stack offers the promise of an understandable arranging, even if it reorders chronological and spatial relations. In 'trompe-l'œil', a mess is always a calculated one, in AR, the reference to the place protects from randomness. If an object and an information appear connected, we imply a logic-however, we imply a unit of meaning that does not necessarily belong to it, just because it is visually bundled.

Furthermore, just imagine that anyone could leave messages with AR or that all available information about a place would be visible simultaneously-the place would be covered by comments. The commented would drown by its comments. This is only a thought experiment-hence, every superimposition includes the possibility of filling up the field of vision, which opposite would be the uncovering. The model of "heaping" is an extreme case of the superimpositioning, which reminds us, that knowledge is not only an accumulation. Nevertheless, there is something constructive 
about this extreme case. Assume that our perception is always occupied: a grid of knowledge through which we interpret the environment (Serres 2010: 74). Hence, AR can make us aware that our way of seeing is superimposed by the already known— to work on its uncovering.

\section{About the reactivation of 'trompe-l'œil' in the age of digital layering}

Layering is a constitutive procedure, which redistributes the way of seeing. If tied to specific techniques and technologies, the sequence of layers begins to shift. The 'velo' as an apparatus, which at first has stretched out a layer in front of the eyes, mediates, theoretically as well as practically, between the 'trompe-l'œil' and AR. Our gaze, confronted with this current field of vision, is forced to consider a new way of thinking and seeing. The image structure of 'trompel'œil' and the construction of the 'velo' are useful to analyze newer procedures of layering and vice versa the newer procedures allow a more precise description of the traditional ones. To conclude this line of thought, I wish to focus on a work of art in which both ways, tradition and innovation, are crossed.

For this essay, I would like to end up with a brief analysis of Laura Owens' untitled diptych from 2015, which is exhibited in the Museum Brandhorst in Munich, Germany (Fig. 6). Laura Owens applies different techniques known from 'trompe-l'œil', which she elegantly transfers into a thinking of the digital. The thesis is that Owens reactivates the 'trompe-l'œil' as a traditional procedure, because it is an adequate analytical tool for digital image culture. Digital images are not welded together with a carrier. Hence, they show a floating weightlessness. One can allocate a carrier to digital images, but in fine arts the invariance, the unchangeable and necessary mutual conditionality of image and carrier is decisive. For example, a painting has a fixed size and this specific size is necessary for its appearance. Very strictly formulated by Henri Matisse, who, therefore, could not even make a sketch of a smaller format than the original: "If I take a sheet of paper of a given size, my drawing will have a necessary relationship to its format. I would not repeat this drawing on another sheet of different proportions" (Flam 1995: 38). 'Trompe-l'œil' is characterized by the fact that it unsettles the alliance between image and carrier. The carrier of 'trompe-l'œil' pretends to be a part of the real environment (a wooden board, a pin board, etc.) instead of being part of the picture. Moreover, 'trompe-l'œil' passes off the figures on the carrier as carriers themselves (a sheet of paper). AR also disguises the carrier, to make the image float and assert it as a true part of the environment. Owens' image production may be described as one that makes use of the congruence and difference of these two procedures to work onto the alliance of image and carrier.

In the diptych, different techniques structure the various levels of the picture plane. Oil, acrylic paint, Flashe Vinyl Paint, charcoal and gesso assemble on the canvas. I will start with the core layer, the newspaper that fills several 'trompe-l'œils' and provides a career in cubism. Owens uses original silkscreen plates from the 1942 Los Angeles Times, which she found in her studio. It thus already begins with an anachronism, which is pursued even further. The technique of screen printing is applied, but then digitally manipulated and blown-up to the size of the canvas $(350.5 \times 264 \mathrm{~cm})$. The blow-up shows a variance of the original as well as it is credible, since the digital newspaper does not have a strict format. The canvases look like two big screens. I call the newspaper a core layer, because there are further levels both in front of and behind it - a membrane stretched on both sides. To speak of a core layer already rises the suspicion that the carrier has been shifted. The picture is not about foreground and background, it is about different layers, with different functions.

The lowest layer in this work is a drawing of thin, grey strokes, which form a landscape on the canvas. They appear as wallpaper that seems to be placed independent of the layers above. As if the drawing has already occupied the background, which is now challenged by the newspaper. However, this conflict is calmed down by the fact that Owens has digitally perforated the newspaper. These remind us of the broken glass. It is not the physical materiality, rather it is its digital surrogate which is cut. Small holes that allow to look through them. Those small holes which structure the 'velo' to organize the space behind. Due to the frontality of the writing, the newspaper marks a solid layer which is impregnated, while the shadows, especially in the cutout parts, expose the layer as being above the background. The newspaper itself is superimposed by apparently gestural brushstrokes, as well as by cut-outs from the newspaper. Some brushstrokes and cut-outs also cast shadows and thus float on another level above the newspaper. Single strokes of color, such as the striking black in the lower left half of the right-hand picture emerge almost haptic and stretch the membrane forward towards the viewer.

I tried to sort three layers in this painting, but this sorting is deceptive, as Owens interweaves the different levels. The superimpositions and procedures have references to each other. Therefore, in the lower right corner of the left picture, single fragments are cut out of the wire netting and depict a pair of eyes next to those of the cats. From the photograph, an elongated shape runs upwards, which continues the digital cut-out above. This cut-out of the newspaper again is behind the newspaper to add a further layer. Moreover, the color gestures, which are highlighted by an artificial shadow, are definitely no longer gestures. 

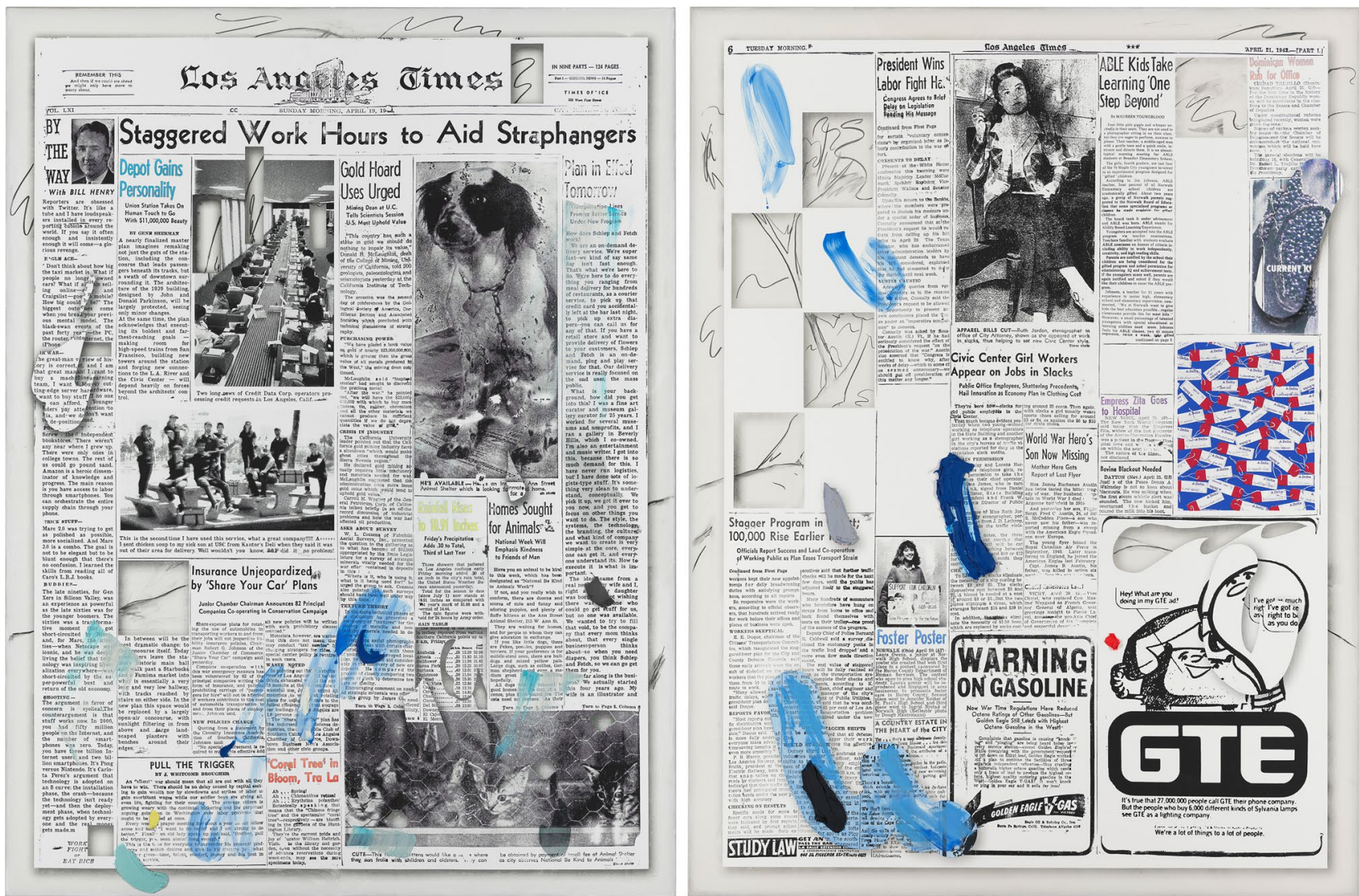

Fig. 6 Laura Owens, untitled, 2015. Oil, acrylic paint, Flashe Vinyl Paint, charcoal and gesso assemble on the canvas. $350.5 \times 264 \mathrm{~cm}$. Collection Museum Brandhorst Munich Copyright: Laura Owens, bpk, Bayerische Staatsgemäldesammlungen, Haydar Koyupinar

They are the result of a planned approach. On the other hand, the impasto applied oil bulges build up a materiality which was just negated in the floating constellation. Finally, Owens introduces blanks into the newspaper and replaces some articles from 1942 with recent or perhaps invented ones. Although there is still much to say about this painting that cannot be fully elaborated here, instead of a layer structure I would like to name three meta-levels.

\subsection{Layering of different production techniques}

Occasionally, reference is made to skeuomorphism in relation to the paintings of Owens (the strategy in which a traditional process is digitally imitated without retaining its function or materiality). The familiar perception makes it easier to handle the new objects. This could mean both the artificial shadow and an artificial impregnation on the screen. In the painting discussed, the layers generate a transfer of various production processes, as well as the transition from imitated to physical materiality. Owens combines digital techniques with traditional ones in one field of vision. What represents information without a carrier in the digital world can appear materially captivated, and what is traditionally associated with a carrier, begins to float on the surface. Different production techniques are displayed and refer to the craftsmanship which ultimately culminate in a representation of these operations. Print, photography, color, drawing, writing, all of them are individual layers and media that mutate into a cipher, each oscillating between the traditional and the digital.

\subsection{The layering of different spatial levels}

The plane of the newspaper draws an inner frame, which can then be crossed by a pasty mass of paint. This overstepping of the inner frame is supported by shadows. Like a staple, the turquoise color mass at the bottom left connects the newspaper to the carrier. Further techniques and layers creep in and remind us of nailing and cutting in traditional 'trompe-l'œil'. Furthermore, there is type, which is traditionally entangled with a carrier and thus supports the materiality of the core layer. Next to the type, however, there are images that burn holes into the solid plane, as 
the digital cuts do. A membrane stretched on both sides without creating an illusion of a physical object. The free drawing behind the newspaper and the brushstrokes that seem to be dancing in the air prevent a comprehensible order. The carrier is pushed forward by the newspaper and simultaneously calmed down by the haptic reality of the color mass.

\subsection{The layering of different temporal levels}

Both the integrated newspaper articles and the different spatial levels allow an anachronistic sequence that is constantly interrupted to continue at another location. At least there is a carryover from the tangle of different layers to the production process, which cannot be clearly traced back. Hence, the modern paradigm of painting to show a transparency of the made as made, is negated, suggesting interchangeability of both, the arranging of layers and of production sequences. Viewing the different layers as being apparently at the same height makes it possible to create new references. Owens' painting can be described as a stack, in which it is never clear which side is at front or which element was placed at the beginning. However, this allows different levels and techniques to be linked. Owens' arrangement is a layernetwork of different relations that can be re-articulated and re-contextualized over and over again, a stack of layers in which each side seems to be connected to each other. As if each layer is represented by a pane of glass and thus has its own background. Each of them is transparent and stands out from the layer below at a real distance, but at the same time, these layers are constantly being penetrated anew. For the view, units of meaning are created when different layers seem to be close to each other, however, with the movement of the eyes the layer structures change and the units of meaning are restacked with them.

The thesis that Owens reactivates the 'trompe-l'œil' as an adequate analytical tool for digital image culture is based on the following overlaps. The 'trompe-l'œil' works into the association between image and carrier in order to undermine its alliance. There is no figure in front of a background, rather every possible figure camouflages itself as a further carrier which appears bundled together by a representation of operations, such as stapling, nailing or gluing. The representation in 'trompe-l'œil' is now linked to the indirectness of digital operations: skeuomorphism, representation of object-oriented tasks, or finally the wellknown representation of touch. In Owens' picture, all these indirect operations are linked. This process, she extends to art-historical operations, like the physical gesture (which absolutely requires a direct physical application of paint). To interrupt directness, a layer, more precisely, a shadow layer, is slid in between. The shadow is, therefore, present before the application of paint. The 'trompe-l'œil' unsettles the carrier, which was never a binding one in the digital. Since 'trompe-l'œil' stacks carrier on carrier, there is no background anymore. A circumstance which Owens transfers to the digital image culture: the newspaper is not the background, instead it appears as a core level from which it can act forward and backwards. The invariance, i.e. the fixed alliance of image and carrier, is decomposed together with a strict sequence of readability, both spatially and temporarily. AR tends to merge technology and reality, as 'trompe-l'œil' tends to embed an image into reality-Owens combines both tendencies, to work at the border between technology, image and reality. The 'trompe-l'œil' in awareness of digital image culture leads Owens to combine and cross information that is traditionally associated with a carrier and information that is not. Both AR and 'trompe-l' œil' are not isolated instances, they want to infiltrate everyday life and everyday perception with the potential to reflect on it.

\section{Summary}

Due to the carrierless appearances that AR throws into space, the 'velo' was questioned as a semi-transparent layer in which the space behind remains visible together with the structure of the grid. The 'velo' as interface is an apparatus of translation, whose materiality and structure, as crucial differences to the window metaphor, become constitutive for the field of vision in 'trompe-l'œil' and in described illustrations. As in AR, the semitransparent layer is used to map something onto it that relates to what is displayed in depth - a mode of thinking and seeing that establishes a layer between the viewer and the visible, commenting on what is seen. This technique can also be found in 'trompel'œil', although the layers are pressed very tightly together so that they unalterably bundle a fixed order. While digital images do not require a specific carrier, AR reintroduces the invariance of placement through the back door. They are not tied to one place and yet they are bound to it by a reference. The 'velo' was built as a translation of depth into flatness, this transfer overlaps the field of vision of AR and thus guarantees the proximity of superimposed information with the object-as if they were connected on one layer. It can superimpose objects-with previous or future versions of this object or with references to other images or objects. A flat object can also be superimposed with a deepening or heightening that transforms spatiality. Also fixed images can be superimposed by motion. Strict orders, whether chronological or in spatial depth, thus become loose and can be restacked. Hence, even these new orders are not fixed, which means that the projection can overlay an object without erasing it as well as it can be removed again without leaving any residue. Since the relationship is not a fixed one, this demonstrates a great potential for open, flexible and variable 
commitment. The information is then also not definite, because the space of information is constantly shifted. Ultimately, classical categories such as background and surface are no longer stable, as they have become interchangeable through digital superimposition. The way of machine seeing in AR recognizes the real environment. To superimpose the real environment means to transform the perception of this environment. The biggest challenge for this new way of seeing is to define the limits and differences of the new field of vision, as these are constantly stretching and become blurred in the process. "Crossroads of seeing" ultimately means to pause at this crossroad, not only to look at the intersection, but also to see where the ways divide.

Acknowledgements Prof. Dr. Carolin Meister, Prof. Dr. Stephan Günzel, Prof. Dr. Matthias Bruhn, Moritz Queisner MA.

Funding Open Access funding enabled and organized by Projekt DEAL. The research project ist funded by FAZIT-STIFTUNG Gemeinnützige Verlagsgesellschaft mbH, Frankfurt.

Availability of data and material Not applicable, separate permissions are required.

\section{Compliance with ethical standards}

Conflict of interest Not applicable.

Open Access This article is licensed under a Creative Commons Attribution 4.0 International License, which permits use, sharing, adaptation, distribution and reproduction in any medium or format, as long as you give appropriate credit to the original author(s) and the source, provide a link to the Creative Commons licence, and indicate if changes were made. The images or other third party material in this article are included in the article's Creative Commons licence, unless indicated otherwise in a credit line to the material. If material is not included in the article's Creative Commons licence and your intended use is not permitted by statutory regulation or exceeds the permitted use, you will need to obtain permission directly from the copyright holder. To view a copy of this licence, visit http://creativecommons.org/licenses/by/4.0/.

\section{References}

Alloa E (2011) Das Durchscheinende Bild. Konturen einer medialen Phänomenologie. Diaphanes, Zurich

Ebert-Schifferer S (2016) Der Durchblick und sein Gegenteil. Malerei als Täuschung. In: Hedinger B, Boehm G (eds) Täuschend echt. Illusion und Wirklichkeit in der Kunst. Hirmer, Munich, pp 16-23

Edgerton S (2004) Giotto und die Erfindung der dritten Dimension. Malerei und Geometrie am Vorabend der wissenschaftlichen Revolution. Wilhelm Fink, Munich

Elcott N (2016) The phantasmagoric dispositif. An assembly of bodies and images in real time and space. Grey Room 62, Winter, pp. 42-71. https://www.columbia.edu/cu/arthistory/faculty/Elcott/ Phantasmagoric-Dispositif.pdf. Accessed 15 Sep 2020

Fahle O (2006) Augmented Reality. Das partizipierende Auge. In: Neitzel B, Nohr R (eds) Das Spiel mit dem Medium Partizipation-Immersion - Interaktion. Schüren, Marbourg, pp 91-103

Flam J (1995) Matisse on art. University of California Press, Berkeley

Friedberg A (2006) The virtual window. From Alberti to Microsoft. The MIT Press, Cambridge

Glaser K, Schröter J (2013) 'Tag that wall'. Augmented Reality-Apps am Beispiel der Street Art zwischen Skripten und Praktiken. Sprache und Literatur 44(1):30-48. https://doi.org/10.1163/25890 859-044-01-90000004

Graw I (2017) The love of painting. Genealogy of a success medium. Sternberg Press, Berlin

Husserl E (2005) Phantasy, image consciousness, and memory (18981925) (trans: John Brough). Collected Works Vol. XI Springer, Dordrecht, Netherlands. https://doi.org/10.1007/1-4020-2642-0

Serres M (2010) Malfeasance. Appropriation through pollution? Stanford University Press, Standford California

Siegert B (2015) Cultural techniques. Grids, filters, doors and other articulations of the real (trans: Geoffrey Winthrop-Young). Fordham University Press, New York

Wagner M (2010) Das zerbrochene Glas. Opake Kommentare auf einem transparenten Medium. In: Hedinger B, Boehm G (eds) Täuschend echt-Illusion und Wirklichkeit in der Kunst. Wilhelm Fink Verlag, Munich, pp 40-47

Publisher's Note Springer Nature remains neutral with regard to jurisdictional claims in published maps and institutional affiliations. 